\title{
Quantitative Surface Coverage Measurements of Self-Assembled Monolayers by Nuclear Reaction Analysis of Carbon-12
}

\author{
Abel T. Demissie, ${ }^{\star}$ Greg Haugstad, ${ }^{\circledR}$ and C. Daniel Frisbie, ${ }^{*}$ \\ Department of Chemical Engineering and Materials Science, University of Minnesota, \\ 421 Washington Ave SE, Minneapolis, MN 55455 \\ " Characterization Facility, University of Minnesota, 100 Union St. SE, Minneapolis, MN 55455 \\ *To whom correspondence should be addressed \\ E-mail: frisbie@umn.edu
}

RBS is a non-destructive technique that is used to measure the elemental composition of a sample. A beam of high energy ${ }_{2}^{4} \mathrm{He}^{2+}$ ions impinges on a sample and the backscattered counts versus energy of the ions are used to calculate the surface coverage and elemental composition according to equation 2. The basic principle that describes RBS is an elastic (hard sphere) collision. From first principles ${ }^{1}$, the kinematic factor $(K)$, which is the ratio of backscattered ${ }_{2}^{4} H e^{2+}$ energy $\left(\mathrm{E}_{1}\right)$ to incident ${ }_{2}^{4} H e^{2+}$ energy $\left(\mathrm{E}_{0}\right)$ is used to calculate the mass of the target atom. The Ortec silicon ion detector was positioned at a scattering angle $(\theta)$ of $165^{\circ}$ with respect to the incident beam and subtended a solid angle of $3.6 \mathrm{msr}$.

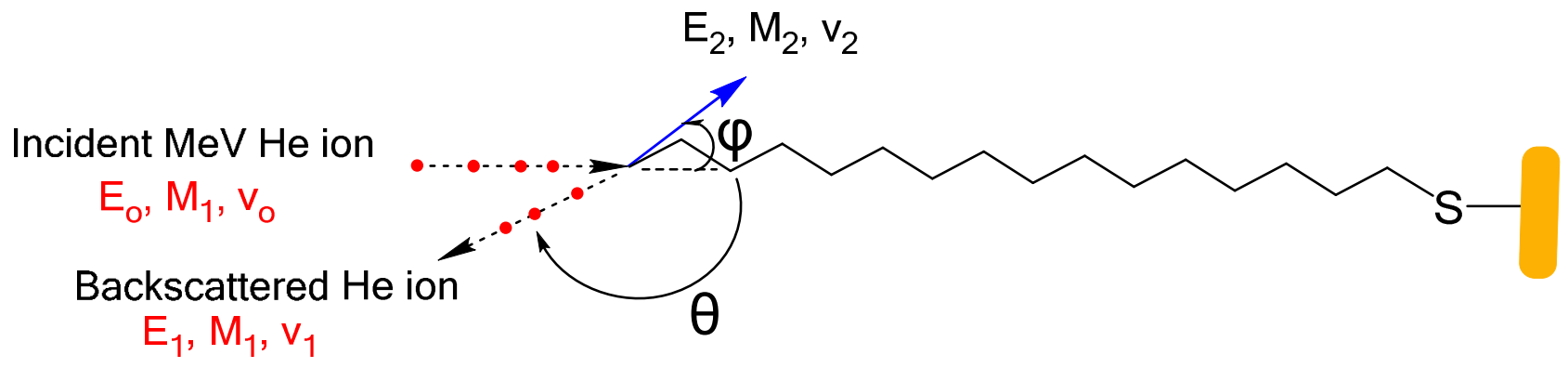




\section{Conservation of Energy}

$\frac{1}{2} M_{1} v_{0}^{2}=\frac{1}{2} M_{1} v_{1}^{2}+\frac{1}{2} M_{2} v_{2}^{2}$

$E_{1}=K E_{0} \quad$ Backscattering
Conservation of Momentum

$$
\begin{aligned}
M_{1} v_{0}= & M_{1} v_{1} \cos \theta+M_{2} v_{2} \cos \phi \\
0= & M_{1} v_{1} \sin \theta-M_{2} v_{2} \sin \phi . \\
& E_{2}=K_{r e c} E_{0} \quad \text { Forward Recoil }
\end{aligned}
$$

Eliminating $\phi$ and $v_{2}$

$$
\begin{array}{ll}
\frac{v_{1}}{v_{0}}=\left[\frac{\left(M_{2}^{2}-M_{1}^{2} \sin ^{2} \theta\right)^{\frac{1}{2}}+M_{1} \cos \theta}{\left(M_{1}+M_{2}\right)}\right] \text { for } M_{1} \leq M_{2} \quad & K_{r e c}=\left[\frac{4 M_{1} M_{2} \cos ^{2} \phi}{\left(M_{1}+M_{2}\right)^{2}}\right] \\
K=\left[\frac{\left(M_{2}^{2}-M_{1}^{2} \sin ^{2} \theta\right)^{\frac{1}{2}}+M_{1} \cos \theta}{M_{1}+M_{2}}\right]^{2} &
\end{array}
$$

Note: In the above equation, phi $(\varphi)$ and theta $(\theta)$ are not the azimuthal and sample normal angles.

Low atomic mass elements, such as ${ }^{12} \mathrm{C}$, that are difficult to detect with RBS because their signal lies spectrally atop the much stronger substrate signal (Rutherford backscattering peak counts being proportional to atomic number squared), and with its correspondingly substantial noise. In NRA, an incident ${ }_{2}^{4} \mathrm{He}^{2+}$ ion may initiate a nuclear reaction by penetrating a target nucleus if it has enough energy to overcome the Coulomb barrier, forming an intermediate excited nuclear state that immediately decays back to the ground state by emission of a particle in the case of prompt reactions. (Some nuclear reactions generate more slowly decaying radioactive states, on the scale of minutes to hours, but we are not accessing such reactions in the present work.) This non-Rutherford scattering process can result in a distinct enhancement of the signal (peak intensity) of target nuclei because the quantum mechanics for activation of such 
nuclear states produces a scattering cross section that is much higher than classical Columbic scattering as shown below.

NRA spectra of alkyl thiols were also collected for SAMs on Au-coated mica and pyrolytic graphite sheet (PGS) substrates as shown in the Figures S2 and S3, respectively. PGS is an ideal substrate for NRA because the ${ }^{12} \mathrm{C}$ signal does not lie atop the substrate signal as in the $\mathrm{Si}$ and mica cases. Instead, the substrate and thiol ${ }^{12} \mathrm{C}$ peaks are located next to each other with a slight tailing of the PGS C peak towards the SAM C peak due to roughness of the PGS. This was corrected by a linear baseline subtraction method. Spectra were collected at 3 different positions, $25 \mu \mathrm{C}$ at each location. The quality of alkyl thiol SAMs on the $\mathrm{Au} / \mathrm{Cr} / \mathrm{PGS}$ substrate was checked with XPS. As shown in Figure S4, the survey spectra of C16 SAMs on $\mathrm{Au} / \mathrm{Cr} / \mathrm{Si}$ and $\mathrm{Au} / \mathrm{Cr} / \mathrm{PGS}$ substrates are similar. Furthermore, the high resolution $\mathrm{C} 1 \mathrm{~s}$ atom count for SAMs on PGS increased with chain length as shown in Figure S5. Collectively, the XPS data indicate that good quality monolayers were prepared on $\mathrm{Au} / \mathrm{Cr} / \mathrm{PGS}$ with reasonable surface coverages. The NRA ${ }^{12} \mathrm{C}$ atom surface coverage was calculated according to equation 2 , and the results are shown in Figure S6. Overall there is good agreement in the coverage values for alkyl thiols on $\mathrm{Au} / \mathrm{Cr} / \mathrm{Si}, \mathrm{Au} / \mathrm{Cr} / \mathrm{mica}$, and $\mathrm{Au} / \mathrm{Cr} / \mathrm{PGS}$. 


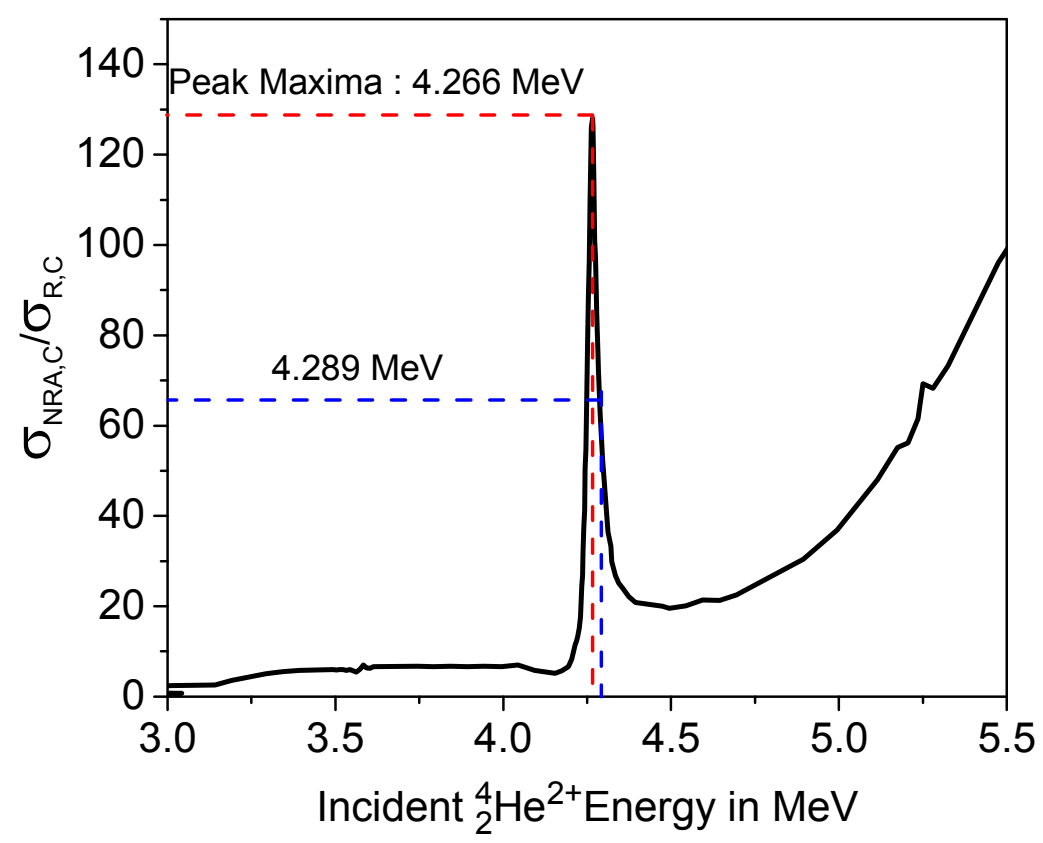

Figure S1. Ratio of nuclear resonant scattering cross section to rutherford scattering cross section of $C$ veres incident beam energy. ${ }^{2}$

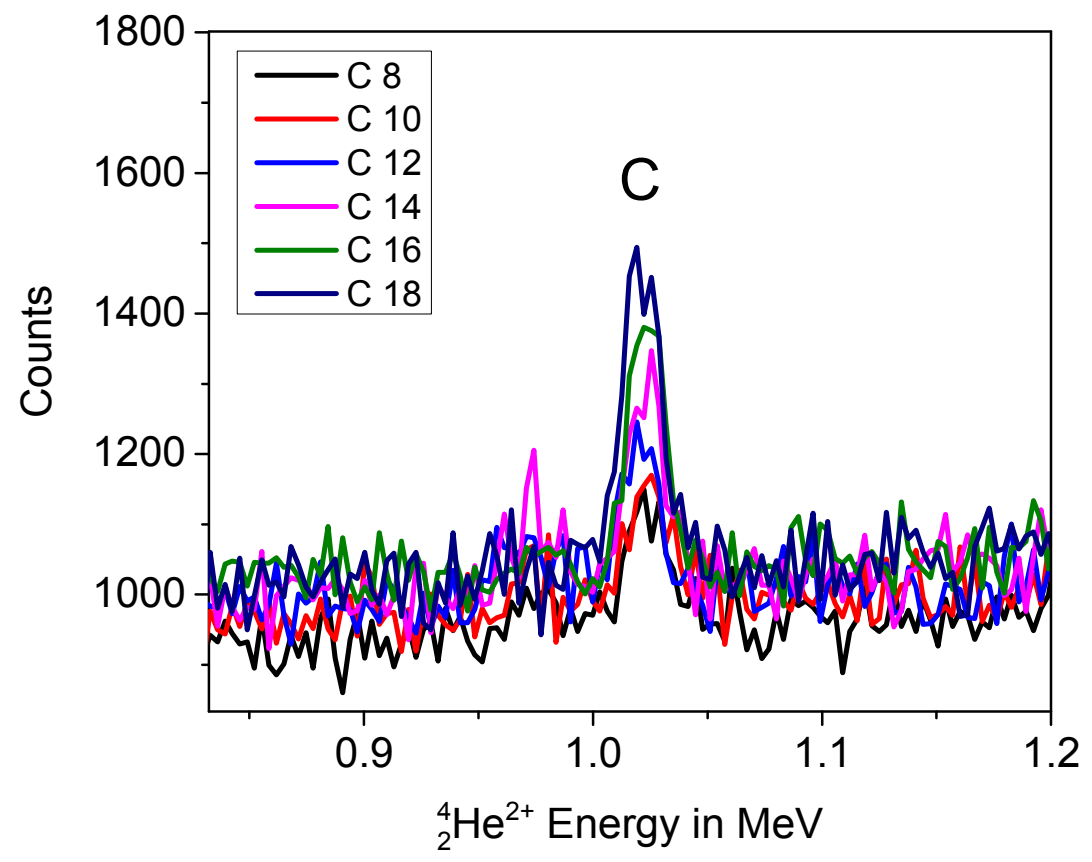

Figure S2. NRA spectra of C8, C10, C12, C14, C16, and C18 thiols on mica (22 min acquisition time per spectrum). The NRA spectra on mica samples showed closer peaks because the dose was smaller $(40 \mu \mathrm{C})$ than SAMs on Si substrates in Figure $1 \mathrm{~b}(75 \mu \mathrm{C})$. The sensitivity to ${ }^{12} \mathrm{C}$ atoms is affected by incident beam energy, and magnitude of resonance scattering cross section as shown in Figure S1. 
A)

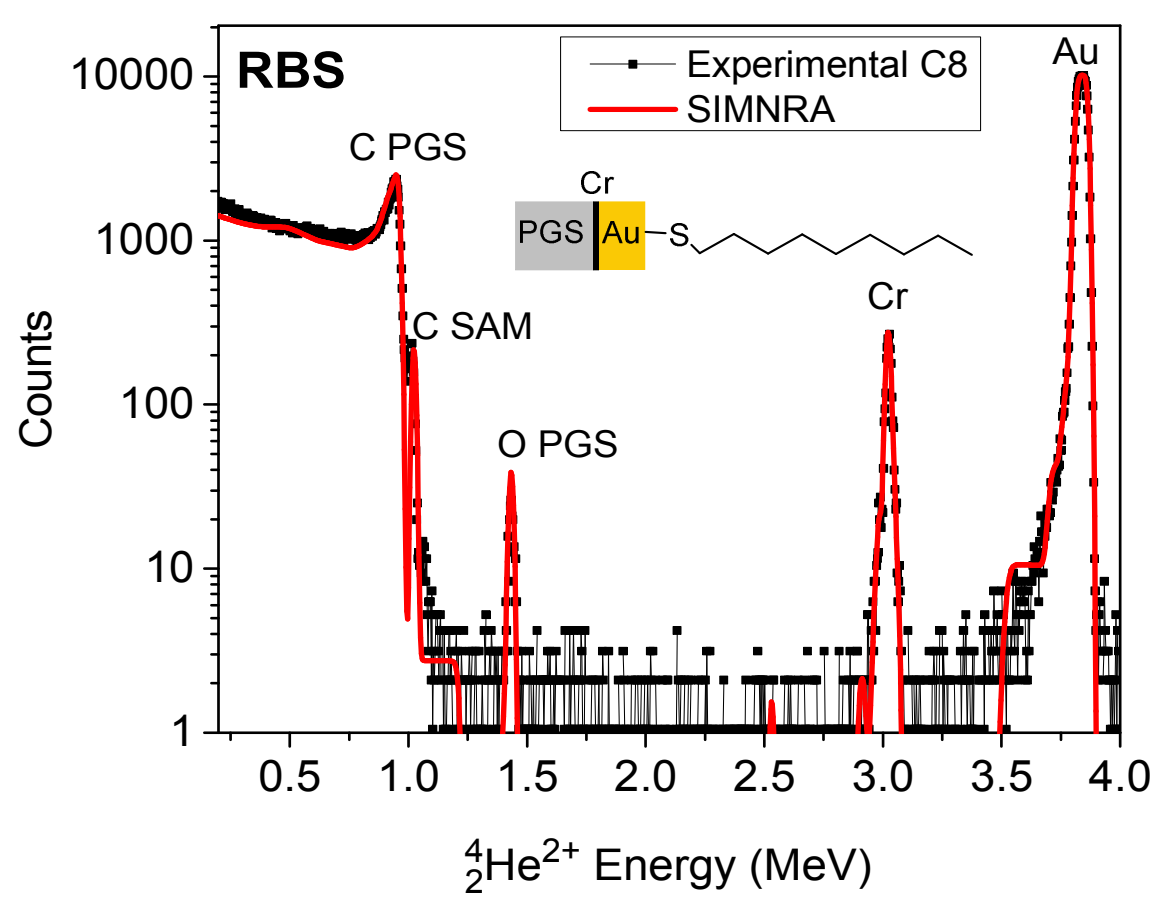

B)

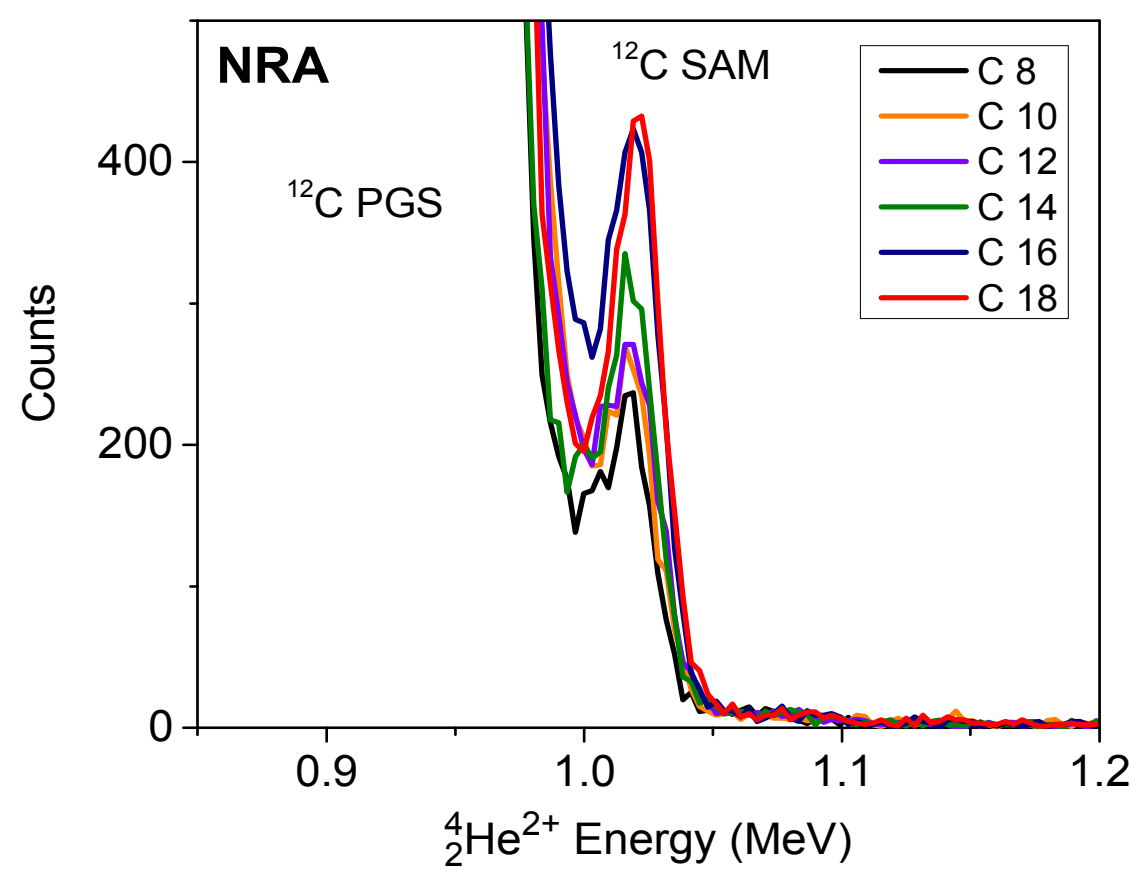

Figure S3. RBS \& NRA spectra of alkyl thiols. A) RBS spectrum of C8 thiol on $50 \mathrm{~nm} \mathrm{Au} \mathrm{/} 5$ $\mathrm{nm} \mathrm{Cr}$ deposited on PGS (pyrolytic graphite sheet) substrate. B) Corresponding NRA spectra of $\mathrm{C} 8, \mathrm{C} 10, \mathrm{C} 12, \mathrm{C} 14, \mathrm{C} 16$, and $\mathrm{C} 18$ thiols (35 min acquisition time per spectrum). 
A)

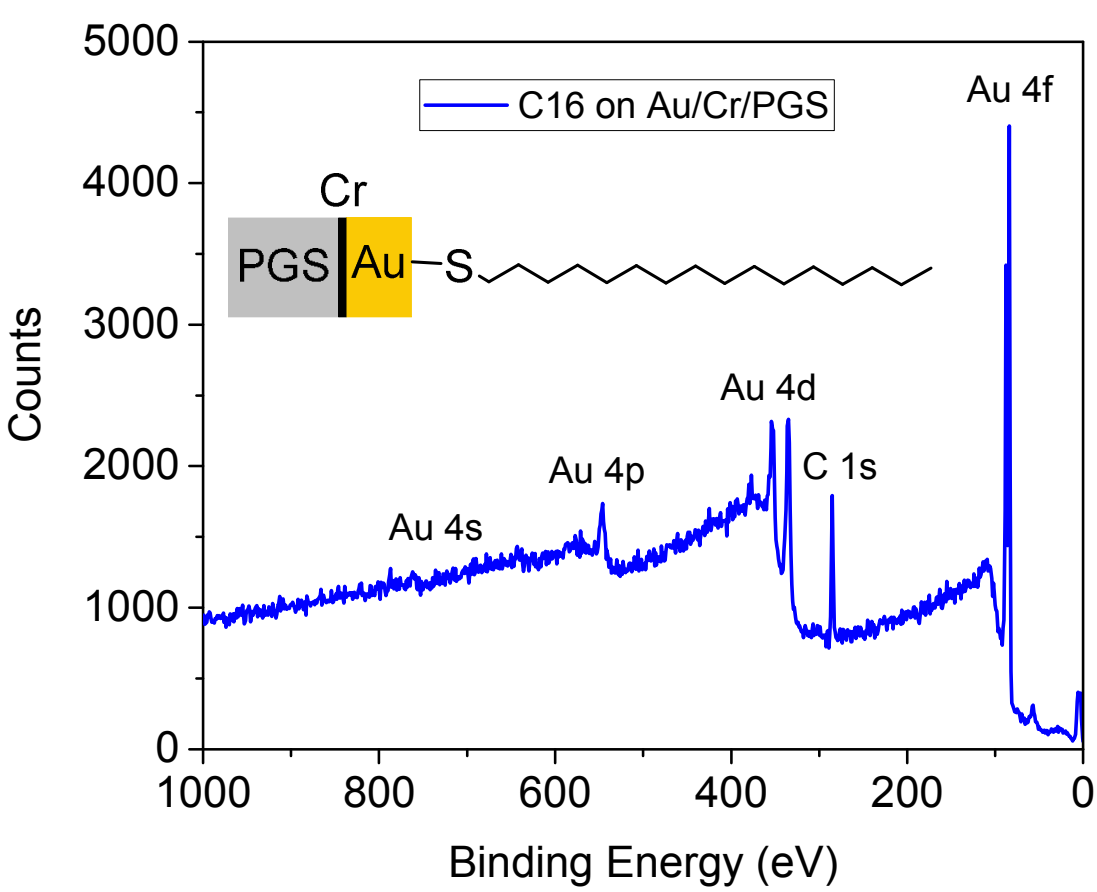

B)

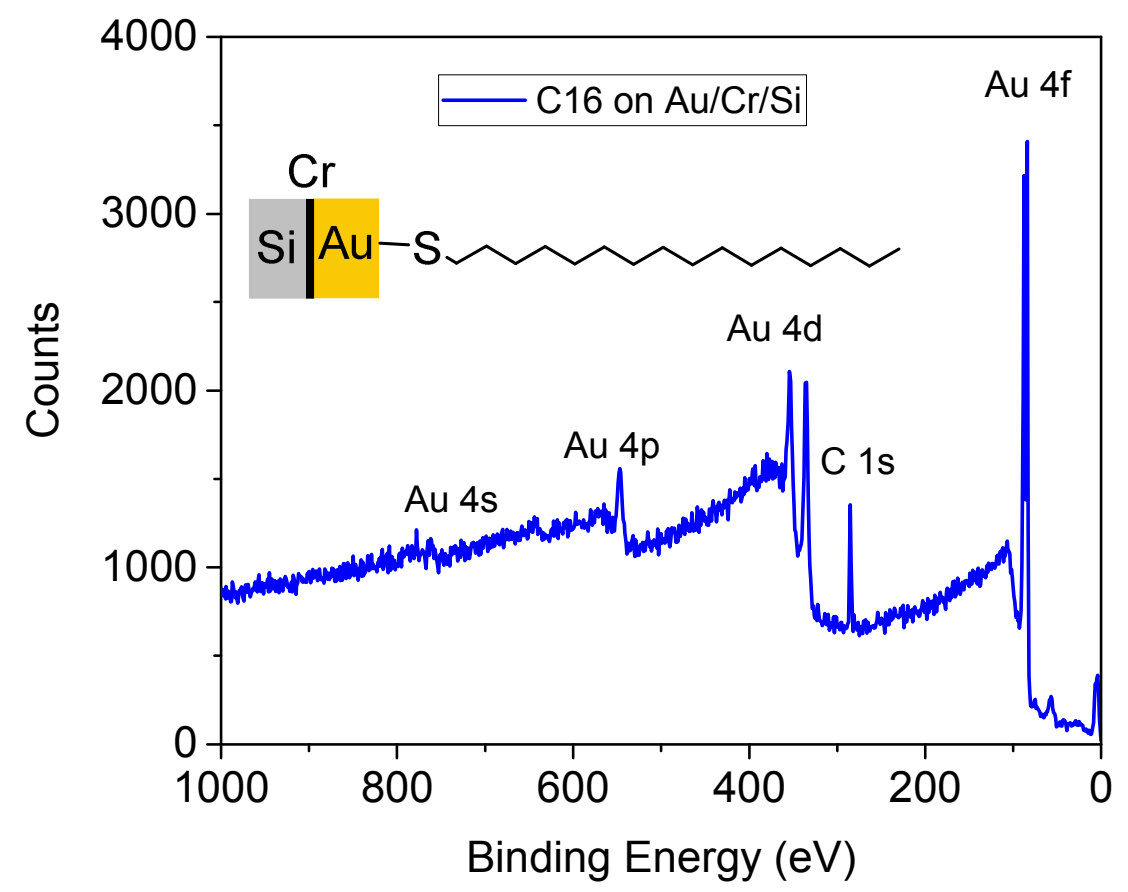

Figure S4. XPS survey spectrum of $\mathrm{C} 16$ on $\mathrm{Au} / \mathrm{Cr} / \mathrm{PGS}(\mathrm{A})$ and on $\mathrm{Au} / \mathrm{Cr} / \mathrm{Si}(\mathrm{B})$. The intensity of $\mathrm{C} 1 \mathrm{~s}$ to $\mathrm{Au} 4 \mathrm{f}$ suggests that good quality films were deposited on the PGS substrate. 


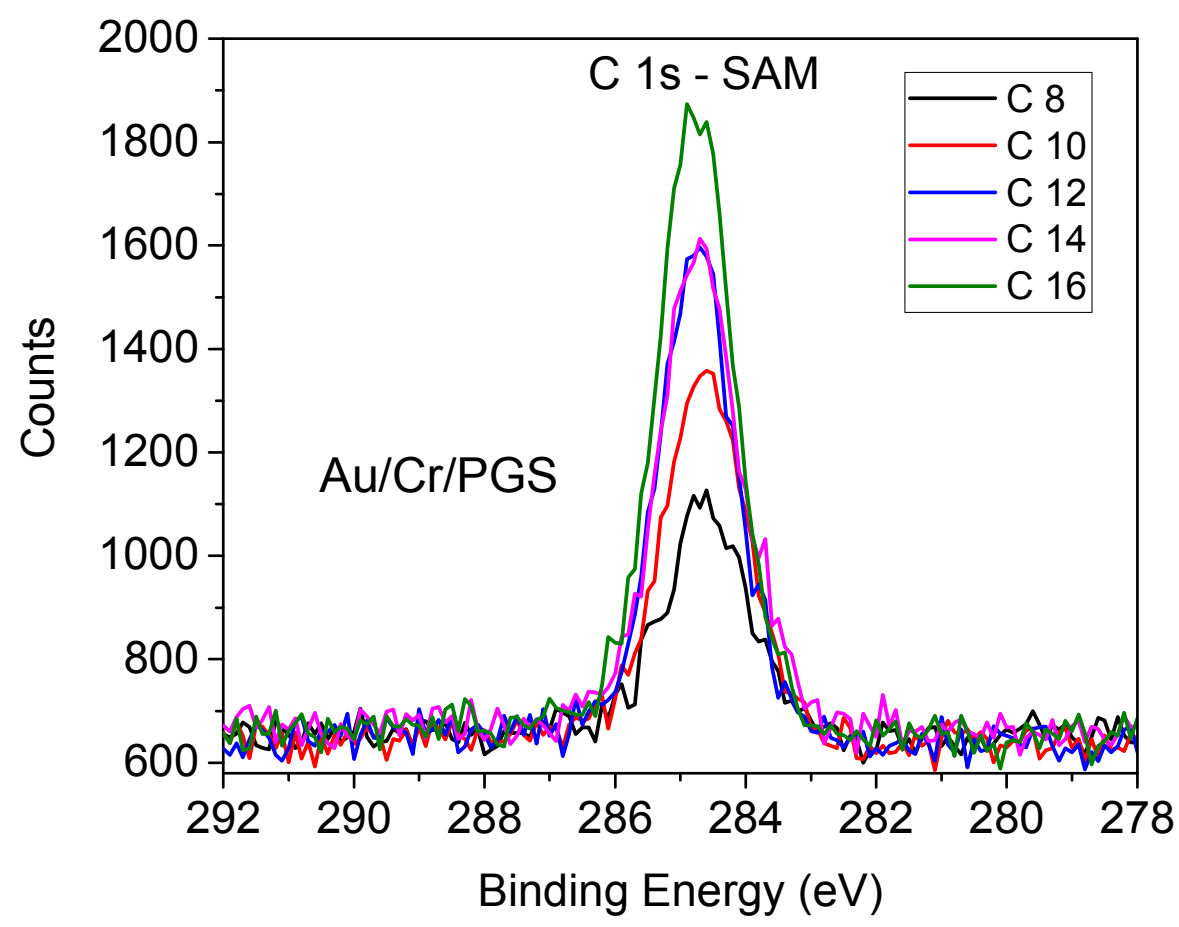

Figure S5. XPS C1s high resolution spectra of C8, C10, C12, C14, and C18 thiols on $\mathrm{Au} / \mathrm{Cr} / \mathrm{PGS}$. As expected, the $\mathrm{C}$ 1s counts increased with the number of $\mathrm{C}$ in each SAM.

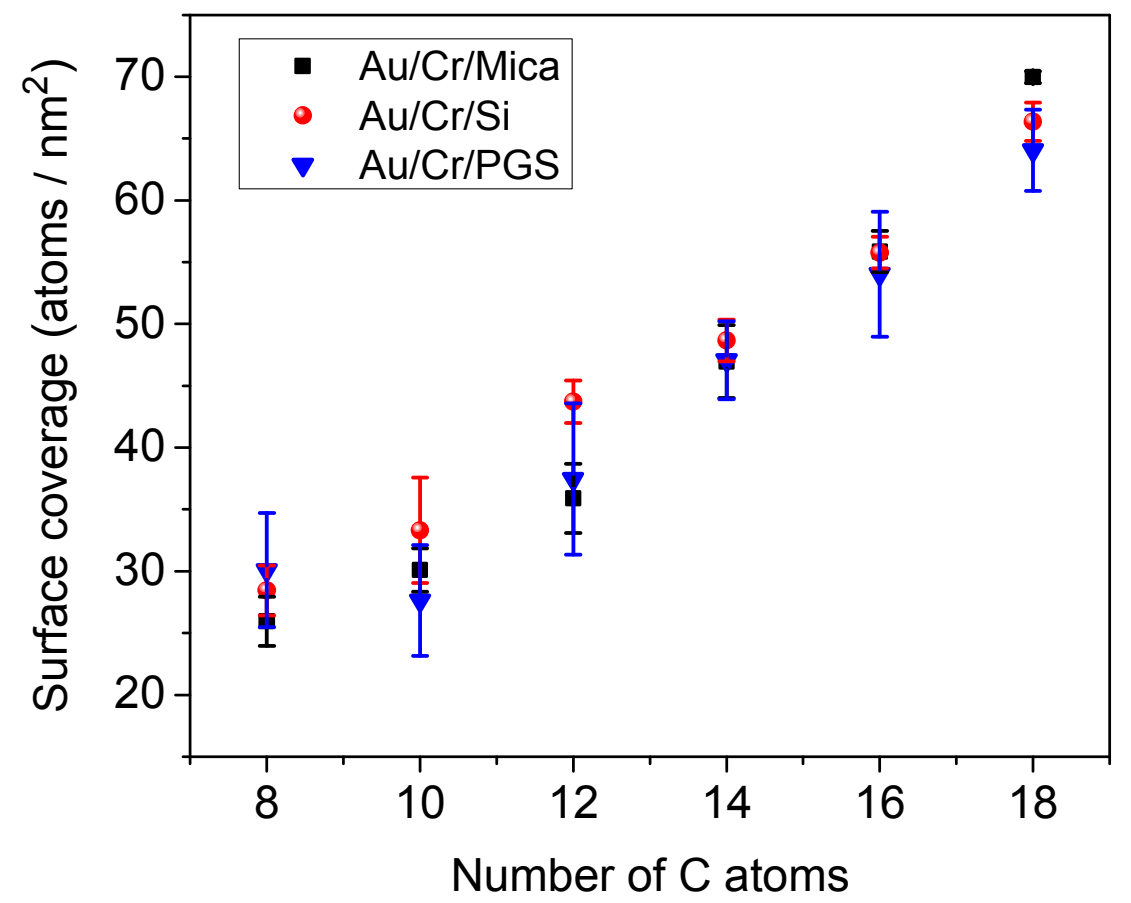

Figure S6. Surface coverage results of alkyl thiols on Au/Cr/Mica (black squares), Au/Cr/Si (red circles), and Au/Cr/PGS (blue triangles).Overall, there is good agreement of coverage values across the different substrates. 


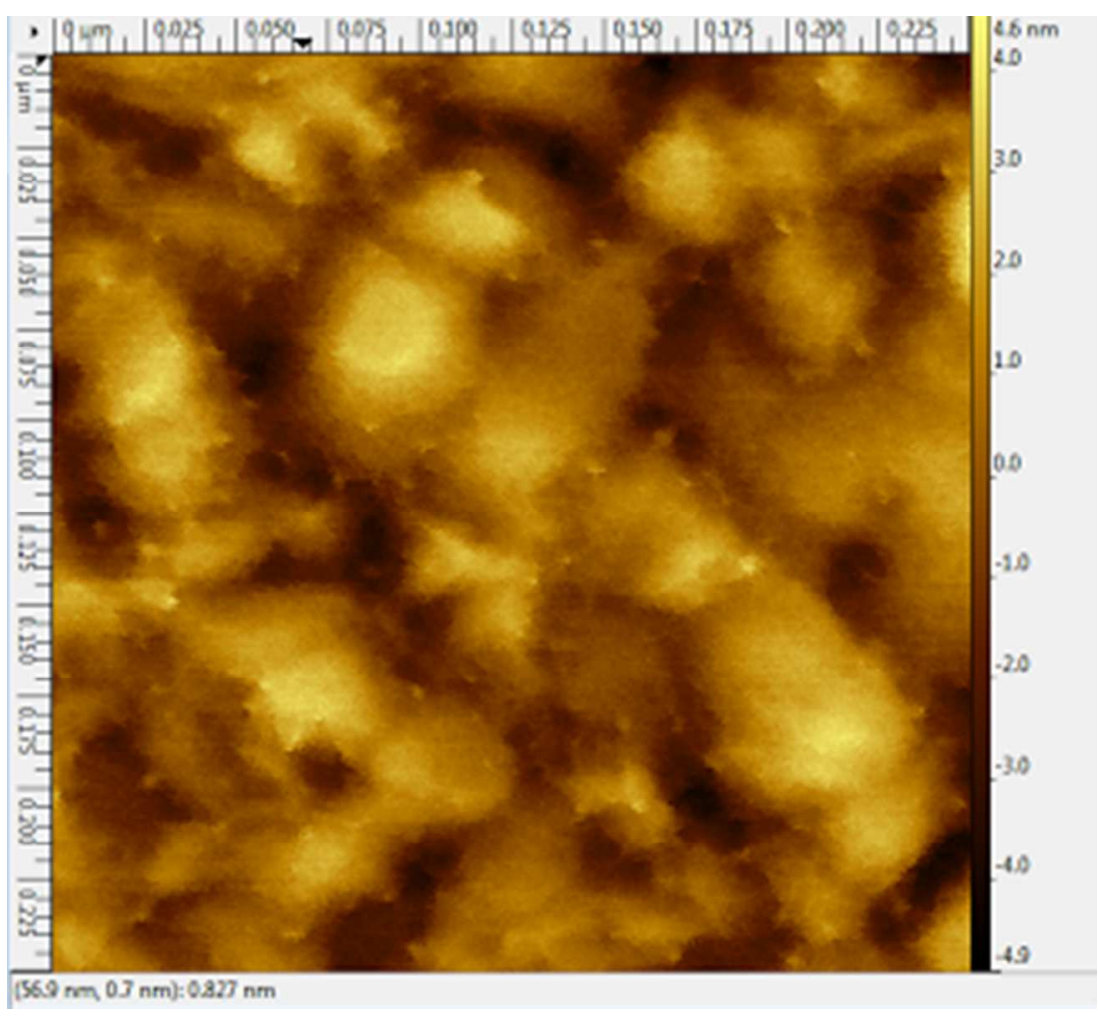

Figure S7. AFM image of bare Au on Au/Cr/Si substrate. Image based on $250 \mathrm{~nm} \times 250 \mathrm{~nm}$ scan size. Excess area 25\%.

\section{References}

(1) Chu, W.-K.; Mayer, J. W.; Nicolet, M. Backscattering Spectrometry; Academic Press, INC: New York, 1978.

(2) Feng, Y.; Zhou, Z.; Zhou, Y.; Zhao, G. Cross Sections for $165^{\circ}$ Backscattering of 2.0-9.0 MeV 4He from Carbon. Nucl. Instruments Methods Phys. Res. Sect. B Beam Interact. with Mater. Atoms 1994, 86, 225-230. 